\title{
Transformation of a Virtual Action Plan into a Motor Plan in the Premotor Cortex
}

\author{
Yoshihisa Nakayama, Tomoko Yamagata, Jun Tanji, and Eiji Hoshi \\ Tamagawa University Brain Science Institute, Tokyo 194-8610, Japan
}

\begin{abstract}
Before preparing to initiate a forthcoming motion, we often acquire information about the future action without specifying actual motor parameters. The information for planning an action at this conceptual level can be provided with verbal commands or nonverbal signals even before the associated motor targets are visible. Under these conditions, the information signifying a virtual action plan must be transformed to information that can be used for constructing a motor plan to initiate specific movements. To determine whether the premotor cortex is involved in this process, we examined neuronal activity in the dorsal premotor cortex (PMd) of monkeys performing a behavioral task designed to isolate the behavioral stages of the acquisition of information for a future action and the construction of a motor plan. We trained the animals to receive a symbolic instruction (color and shape of an instruction cue) to determine whether to select the right or left of targets to reach, despite the physical absence of targets. Subsequently, two targets appeared on a screen at different locations. The animals then determined the correct target (left or right) based on the previous instruction and prepared to initiate a reaching movement to an actual target. The experimental design dissociated the selection of the right/left at an abstract level (action plan) from the physical motor plan. Here, we show that activity of individual PMd neurons initially reflects a virtual action plan transcending motor specifics, before these neurons contribute to a transformation process that leads to activity encoding a motor plan.
\end{abstract}

Key words: premotor; frontal; motor cortex; motor control; sensorimotor; macaque

\section{Introduction}

The premotor cortex (PM) of primates is thought to play an important role in sensory guidance of motor behavior (Wise, 1985; Mushiake et al., 1991; Kalaska and Crammond, 1992; Passingham, 1993; Caminiti et al., 1998). For visual guidance of actions, at least two mechanisms for visuomotor guidance have been proposed; one is a process involving the transformation of visuospatial information about motor targets into information specifying motor parameters using body-centered coordinates (standard visuomotor mapping), whereas the other is an arbitrary association between a sensory signal and a movement (nonstandard mapping) (Caminiti et al., 1991; Crammond and Kalaska, 1994; Murray et al., 2000; Wise and Murray, 2000). For the latter, the involvement of the PM in learning and executing the association of a visual object characterized by a specific color or form with a particular movement has been extensively studied (Petrides, 1982, 1986; Halsband and Passingham, 1985; Canavan et al., 1989; Halsband and Freund, 1990; Mitz et al., 1991; Bous-

\footnotetext{
Received May 25, 2008; revised Aug. 21, 2008; accepted Aug. 25, 2008.

This work was supported by Grants-in-Aid for Scientific Research on Priority Areas (Integrative Brain Research) from the Ministry of Education, Culture, Sports, Science, and Technology of Japan (MEXT) 18019032 and 20019027 (E.H.) and 16067101 (J.T.), Grant-in-Aid for Japan Society for the Promotion of Science (JSPS) Fellows 20-9883 (Y.N.), Grants-in-Aid for Young Scientists from MEXT (A) and from JSPS (S) 18680035 and 19670004 (E.H.), and a Career Development Award from the International Human Frontier Science Program Organization (E.H.). We are deeply grateful to Dr. B. J. Richmond for his insightful comments on this work. We thank members of the Tanji Laboratory for assistance and discussions and S. Hoffman for technical assistance.

Correspondence should be addressed to Dr. Eiji Hoshi, Tamagawa University Brain Science Institute, Tamagawa Gakuen 6-1-1, Machida, Tokyo 194-8610, Japan. E-mail: hoshie@lab.tamagawa.ac.jp.

D0I:10.1523/JNEUROSCI.2372-08.2008

Copyright $\odot 2008$ Society for Neuroscience ～0270-6474/08/2810287-11\$15.00/0
}

saoud and Wise, 1993; di Pellegrino and Wise, 1993; Kurata and Hoffman, 1994; Deiber et al., 1997; Asaad et al., 1998; Grafton et al., 1998; Toni et al., 2001; Eliassen et al., 2003; Boettiger and D'Esposito, 2005). However, there are cases in which the selection of an action is achieved even in the absence of either standard or nonstandard mapping. For instance, instructions calling for the selection of action may be given as verbal or nonverbal commands in the absence of motor targets. Under this type of condition, instructions conveying such demands as "move right" or "pick up an object that will appear on the left" often provide partial information about future actions and do so at different levels of abstraction; no visual information is available to guide motor selection. We refer to the type of action determined at this conceptual level as virtual action. Subsequently, the virtual action is transformed, often with an intervening interval, into an actual motor plan when an action target is physically provided. Consideration of these two-step processes led us to examine whether the PM represents the action at this conceptual level and whether the $\mathrm{PM}$ is involved in the transformation of the virtual action into a motor plan.

To address these issues, we used an experimental model in which an instruction to reach for a future target given at a conceptual level was followed by the presentation of an actual target. We analyzed cellular activity in the dorsal PM (PMd) of monkeys while they were engaged in a behavioral task that required the transformation of a virtual action into a motor plan when visual signals were used as instructions for the action. We show that a subset of the cellular activity in the PMd first represents the virtual action, which, in turn, is transformed into activity representing the motor plan. 


\section{Materials and Methods}

Animals and experimental conditions. We used two monkeys (Macaca fuscata; one male weighing $6.0 \mathrm{~kg}$ and one female weighing $5.0 \mathrm{~kg}$ ) that were cared for in accordance with the National Institutes of Health guidelines and the guidelines of our institute. During the experimental sessions, each monkey sat in a chair with its head and left arm restrained. We installed a push button in front of the chair at waist level. A 19 inch video monitor equipped with a touch-sensitive screen was placed in front of the monkey. Eye positions were monitored at $240 \mathrm{~Hz}$ with an infrared eye-tracking system (resolution, $0.25^{\circ}$ in visual angle; RHS-M; Applied Science Laboratories). Neuronal activity was recorded with glassinsulated Elgiloy-alloy microelectrodes $(0.5 \sim 2 \mathrm{M} \Omega$ at $1 \mathrm{kHz})$, which were inserted through the dura mater using a hydraulic microdrive (MO81-S; Narishige). Single-unit potentials were amplified with a multichannel processor and sorted using a multispike detector (MCP Plus 8 and MSD; Alpha Omega Engineering). Muscle activity was recorded with stainless-steel wire electrodes. The electromyographic activity was amplified, A-D converted, and stored. We monitored activities in the following muscles during task performance: the biceps and triceps brachii, deltoid (anterior, lateral, and posterior heads), trapezius, supraspinatus, infraspinatus, pectoralis major, rhomboid major, and neck and paravertebral muscles. The TEMPONET system (Reflective Computing) was used to control the behavioral task and save data for off-line analyses at 1000 $\mathrm{Hz}$.

After completion of physiological recordings, we applied intracortical microstimulation (ICMS) through the tip of each inserted electrode (11-44 pulses; $200 \mu$ s width at $333 \mathrm{~Hz}$; current, $<50 \mu \mathrm{A}$ ) and checked sensory response properties of neurons to somatosensory and visual stimuli. In this study, we operationally defined the primary motor area (MI) as the area in which ICMS evoked limb movements or muscle twitches (with $>50 \%$ probability) with currents $<40 \mu \mathrm{A}$ with 11 pulses. At the caudal end of the dorsal aspect of the premotor cortex, we observed that forelimb movements were evoked when 44 trains of ICMS were applied. However, we did not elicit saccadic eye movements at any sites where we sampled neuronal activity for this report, suggesting that the recording sites were outside the rostral part of PMd or the frontal eye field where saccadic eye movements can be evoked with the ICMS (Bruce et al., 1985; Fujii et al., 2000). Thus, the surveyed area corresponds to the caudal division, or area F2, of the dorsal premotor area (PMd) (Matelli et al., 1985; Picard and Strick, 2001), which has been shown to control motor behavior with the arm and shoulder (Fujii et al., 2000; Hoshi and Tanji, 2006, 2007).

Behavioral task. We devised a behavioral task for the monkeys in which three behavioral phases were separated in time (see Fig. $1 \mathrm{~A}$ ): retrieval of a virtual action plan from visual cues, transformation of the virtual action plan into a motor plan, and execution of the motor plan. After an intertrial interval of $\geq 3 \mathrm{~s}$, each trial commenced when the monkey pushed the button with the right hand and gazed at a fixation point (white circle, $1.4^{\circ}$ in visual angle) that appeared at the center of the touch-sensitive screen. If the monkey maintained fixation on the fixation point for $1200 \mathrm{~ms}$, an instruction cue $\left(11^{\circ}\right)$ was presented for $800 \mathrm{~ms}$ to tell the animals to select either the right or left target. At this stage, no information about the future targets was available. The monkeys were required to select right or left, based on the color and shape of the instruction cue. For monkey 1, a green circle or yellow square instructed the monkey to select the target on the right, whereas a red diamond or blue cross indicated that the left target should be selected (see Fig. $1 B$ ). For monkey 2, a green circle and a red diamond specified the selection of the right and left target, respectively. These cues were presented randomly. If the monkey fixated for $1200 \mathrm{~ms}$ during the subsequent instructed delay period, a choice cue consisting of two gray squares (size of each box, $7^{\circ} ; 11^{\circ}$ apart between the centers) appeared at one of six different locations on the screen (see Fig. $1 C)$. After a delay period of $1500-2500 \mathrm{~ms}$, the color changed from gray to white, which served as the GO signal. If the monkey reached for the target with the right arm within the next $1.5 \mathrm{~s}$, it received a fruit juice reward $500 \mathrm{~ms}$ after touching the correct square. The monkey was required to gaze at the choice cue for 300-600 ms before the GO signal appeared.

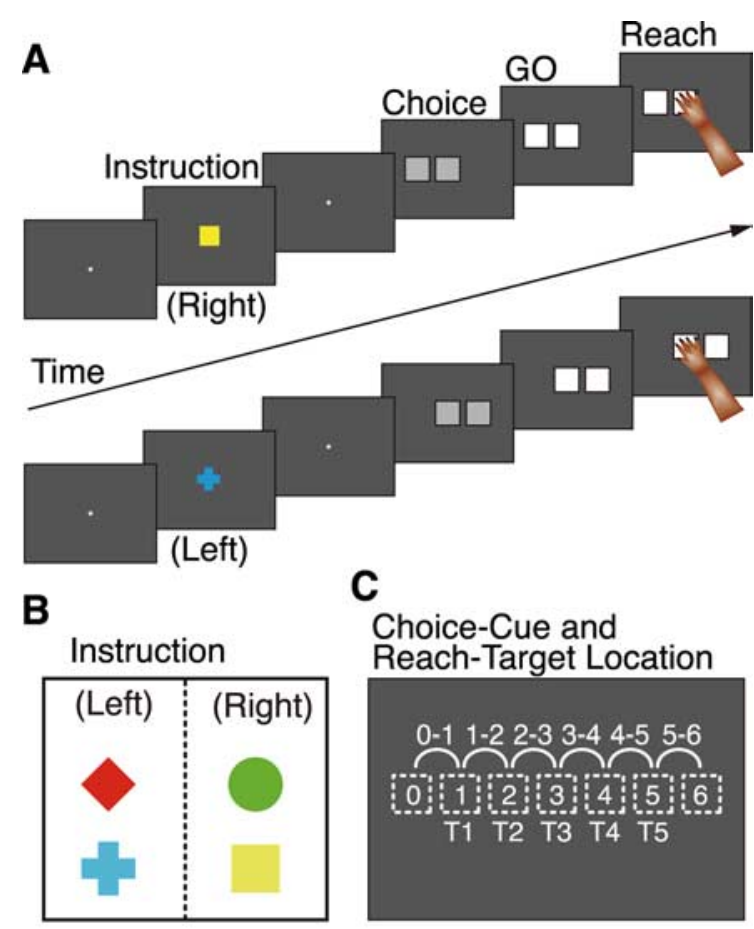

D

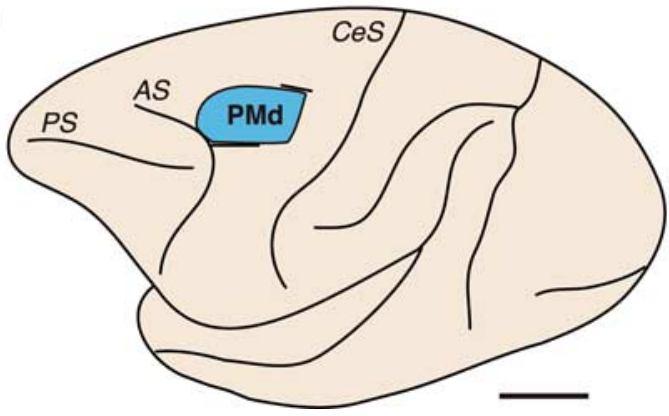

Figure 1. Behavioral task, instruction and cue signals, and the recording site. $\boldsymbol{A}$, The temporal sequence of the behavioral events. $\boldsymbol{B}$, Visual signals used to instruct the selection of the left or right forthcoming choice cue. $\boldsymbol{C}$, Locations of the choice cue and target on the screen. For the choice cue, two gray squares appeared at neighboring positions (locations $0-6$ depicted with dotted squares). The target position was selected from five potential targets (T1-T5), which were either the left or right component of the choice cue. T3 was located at the center of the screen, aligned with the center of the monkey's body. D, A cortical map of the recording site. Neurons from the caudal PMd were analyzed (colored in blue). PS, Principal sulcus; AS, arcuate sulcus; CeS, central sulcus. Scale bar, $10 \mathrm{~mm}$.

The following three points were essential in designing the behavioral task: (1) On receiving the instruction cue, monkeys were able to plan to reach right or left at a conceptual level (virtual action plan), without specifying actual direction of the reach; (2) the determination of where to reach on the screen (formation of a motor plan) was possible for the first time after the appearance of the choice cue; (3) the target position was randomly selected from one of five potential positions on the screen (T1 to T5 in Fig. $1 C$ with T3 at the center of the screen), regardless of the instruction cues. Thus, the selection of right/left cued by the instruction resulted in no bias for spatial attention or the direction of the future reach.

Data analysis. We counted the number of spikes from each neuron in successive $200 \mathrm{~ms}$ bins centered at four task events: the instruction cue onset ( 10 bins; 4 before, 1 around, and 5 after the onset), the choice cue onset (10 bins; 4 before, 1 around, and 5 after the onset), the GO signal onset ( 5 bins; 4 before and 1 around the onset), and the movement onset ( 5 bins; 2 before, 1 around, and 2 after the onset). We classified a neuron 


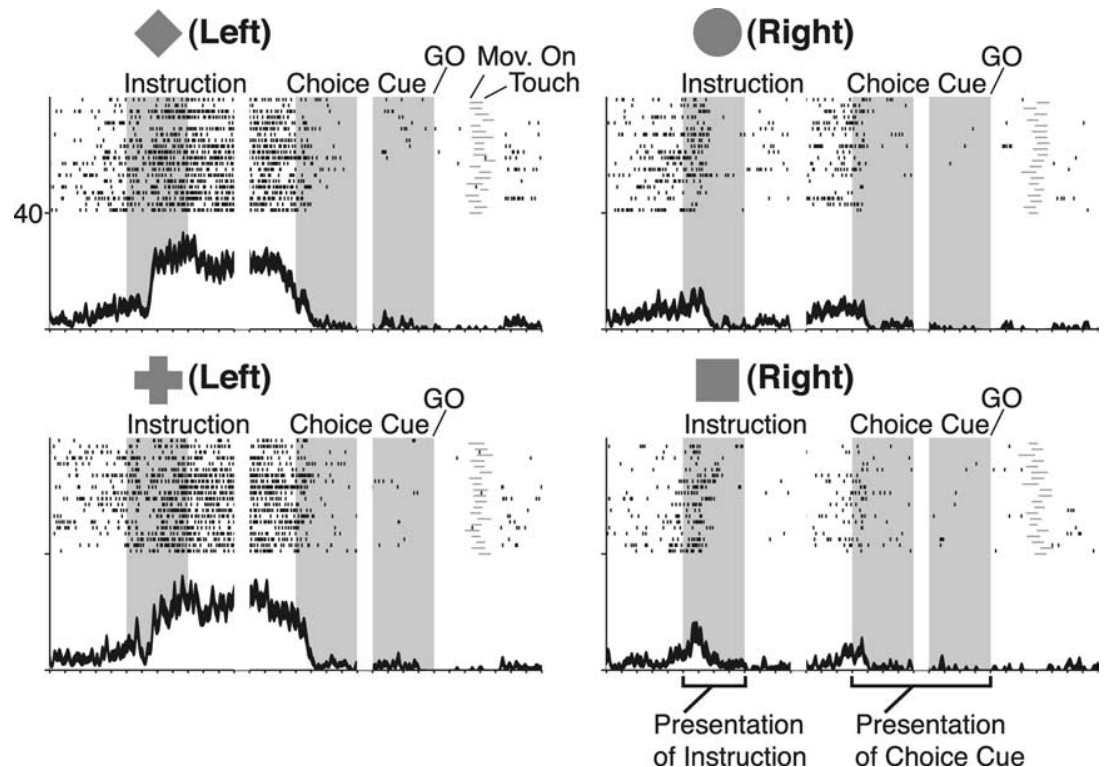

Figure 2. Neuronal activity reflecting the virtual action plan. Activity of this PMd neuron increased when either a red diamond or blue cross was used to specify that the future action was to select the left target, which was not yet visible. Rasters and spike density functions (smoothed using a Gaussian kernel; $\sigma=10 \mathrm{~ms}$; mean $\pm \mathrm{SE}$ ) indicate activity in trials sorted according to four visual signals that instructed the monkey to select either the left or right future target. The ordinate represents the instantaneous firing rate (in spikes/second). Neuronal activity is aligned to the onset of the instruction cue, choice cue, and G0 signal. The gray areas indicate when the instruction cue or choice cue was presented. The tick marks on the horizontal axis are placed at $200 \mathrm{~ms}$ intervals.

as "task-related" if the distribution of the discharge rate (in spikes/second) was significantly different in at least one of the 10 trial types (five positions of the target on the screen, T1 to T5 in Fig. $1 C$, each of which could be associated with a left or right target; ANOVA, $p<0.001$, not corrected). All of the neurons in the database $(n=773)$ were confirmed to be task-related and were sampled in $\geq 50$ trials.

For the spike count data in the $200 \mathrm{~ms}$ bins, we first applied two-way ANOVA looking at the action plan (i.e., left or right side of the choice cue) and the motor plan (i.e., the five target positions on the screen). Based on this analysis, we classified the activity of the neurons into four categories: (1) only action plan selective (action plan, $<0.01$; motor plan, $\geq 0.01$; and action plan by motor plan, $\geq 0.01$ ); (2) only motor plan selective (action plan, $\geq 0.01$; motor plan, $<0.01$; and action plan by motor plan, $\geq 0.01$ ); (3) both selective (action plan, $<0.01$; motor plan, $<0.01$; or action plan by motor plan, $<0.01$ ); and (4) nonselective (action plan, $\geq 0.01$; motor plan, $\geq 0.01$; and action plan by motor plan, $\geq 0.01$ ). Neuronal activity classified as both could play a role in representing the location of the choice cue or in integrating it with the action plan. To examine these possibilities, we applied a second two-way ANOVA to the neuronal activity classified as both, which examined two main factors: the choice cue location and the action plan. For this analysis, we used the data obtained with the cues at positions $1-5$ in Figure $1 C$. We excluded the data of trials in which the choice cue included the position 0 or 6 (see Fig. $1 C$ ) that was never used as a reach target; the information on the action plan was not essential in these trials. The second analysis resulted in the identification of two classes of activity: (1) activity selective only for the choice cue location (choice, $<0.01$; action plan, $\geq 0.01$; and choice by action plan, $\geq 0.01$ ) and (2) activity selective both for the choice cue location and the action plan (choice, <0.01; and action plan, $<0.01$ ). Through the two sets of ANOVA, the activity was classified into five categories: (1) selective only for the action plan, (2) selective only for the motor plan, (3) selective only for the choice cue location, (4) selective for the choice cue location and the action plan (mixed selectivity), and (5) selective for neither.

To estimate how well the neuronal activity reflected the action plan and the motor plan, we calculated a variable termed partial eta squared $\left(\mathrm{hp}^{2}\right)$, using values obtained from the ANOVA table and the following formula: $\mathrm{hp}^{2}=\left(\mathrm{SS}_{\text {effect }}\right) /\left(\mathrm{SS}_{\text {effect }}+\mathrm{SS}_{\text {error }}\right)$. We calculated this variable for each of the two main effects (i.e., the action plan and the motor plan) and for every $200 \mathrm{~ms}$ bin of spike data. $\mathrm{SS}_{\text {effect }}$ is the sum of squares of the main effect. $\mathrm{SS}_{\text {error }}$ is the sum of squares of the error term. This value becomes progressively smaller as the variance becomes more dependent on the error term.

\section{Results \\ Database of neuronal activity recorded from the caudal PMd}

While the monkeys were performing the behavioral task, we recorded neuronal activity from the PMd medial to the genu of the arcuate sulcus (Fig. 1D) (see also Fig. 10). We monitored activity of every neuron that we encountered. If a neuron showed task-related activity, we stored the data sampled for off-line analysis. Approximately 25\% of all PMd neurons whose activity was monitored on-line were found statistically to be task-related. The database for this report included 773 taskrelated neurons (330 in monkey 1 and 443 in monkey 2). We pooled data from the two animals because the selectivity was qualitatively similar between the subjects. In this report, we analyzed the neuronal activity that occurred before the appearance of the GO signal, whereas activity in the muscles analyzed did not show any consistent task-related changes. Because success rates recorded during the behavioral task exceeded $95 \%$ for both monkeys, we focus on neuronal response properties in successful trials.

\section{Neuronal activity reflecting a virtual action plan}

We found that the activities of a subset of the 773 task-related PMd neurons exhibited differential responses to the instruction cues that indicated the selection of the right or left of future targets. A representative example of such neuronal activity is shown in Figure 2, which showed increased activity if a red diamond or a blue cross appeared as the instruction cue. However, the activity was suppressed if a green circle or a yellow square was presented. To examine whether this selectivity reflected the identity of the objects used as instruction cues or the action plan represented by the cue (reach left or reach right), we applied ANOVA with two factors (identity of the objects and the instruction represented by the object; identity was nested in instruction) and a dependent variable (the average discharge rate starting 101 ms after the instruction onset until the choice cue onset). We found that 339 of the 773 neurons (44\%) showed significant differences in their activities based on the instruction provided by the cue (left or right; $p<0.01$ ). Among the 330 neurons that we tested using all four of the visual objects (Fig. $1 B$ ), we found that the activity of only 9 neurons (3\%) exhibited a significant relationship to the cue identity $(p<0.01)$. Thus, the responses to the cues reflected the selection of the right or left target even if the actual targets were absent (i.e., the selection of a forthcoming action in advance of specification of a physical motor plan). These analyses suggested that the neuronal activity signified a virtual action plan (Fig. 2). Notably, activity at this stage does not include information concerning motor variables that are necessary to plan and execute the actual motion because the choice cue was not presented yet. 
Neuronal activity reflecting a motor plan

After the appearance of the choice cue, the action plan selectivity generally declined or ceased. Instead, we observed neuronal activity that was different from the activity described above. The first type of activity started by the time the monkey initiated the reaching movement, and the activity was dependent on the future position of the reach target. In the example shown in Figure 3, the activity during the later part of the choice cue period markedly differed depending on the target location on the screen; it was greater when the choice cue prompted the monkey to plan to capture a target placed on the right side. In most cases, this activity continued to be elevated until the initiation of a movement toward the target. We performed twoway ANOVA to examine the relationship between neuronal activity during the choice cue period and either the target position or the action plan to select right or left (see Materials and Methods). We found that, in $\sim 15 \%$ of the task-related neurons, the activity was dependent on the reach target position, but not on the instruction to reach for the target on the right or left. This type of activity, which appeared after the appearance of the choice cue and before the movement initiation, was judged to be essential for planning to reach for the individual target. We, therefore, determined that this target-selective activity reflected a motor plan.

\section{Neuronal activity reflecting both the action plan and the location of the choice cue}

The second type of neuronal activity observed during the choice cue period was activity that reflected both the action plan and the location of the choice cue. In the example shown in Figure 4, the activity differed depending on whether the monkey was instructed to reach for the right or left target. In addition, the activity also reflected the location of the choice cue; it was greater for choice cues positioned on the right side of the display. The two-way ANOVA applied to the data collected after the appearance of the choice cue revealed a significant relationship of the neuronal activity to both the instruction to select a right or left target and the location of the choice cue (see Materials and Methods). The identification of this type of activity suggested that these neurons were involved in encoding the action plan and the location of the choice cue, both of which were necessary to construct a motor plan. Our observation of neuronal activity representing multiple types of information prompted us to examine, in detail, the time course of the development of the neuronal activities reflecting the action plan, choice cue location, and motor plan.

Time course of the distribution of neurons representing the action plan, choice cue location, and motor plan

To analyze the time course of the distribution of neurons representing each of the behavioral factors, we examined the activities
(Left) Instruction Choice Cue GO
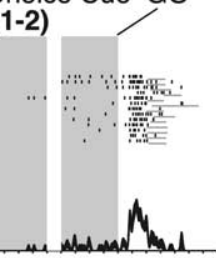

(2-3)

(2-3)

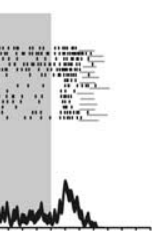

(3-4)

T3

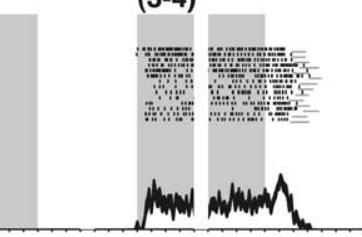

(4-5)
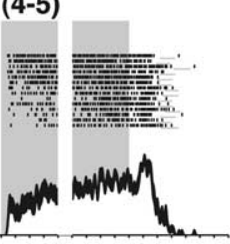

(5-6)

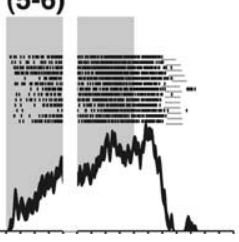

(Right)

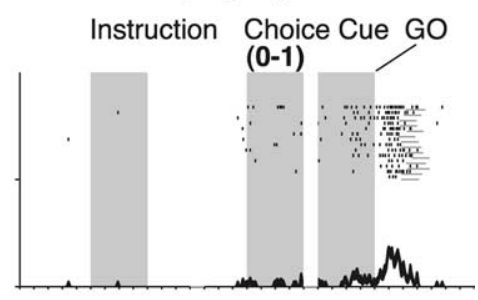

(1-2)

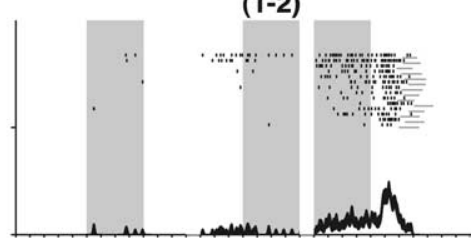

(2-3)

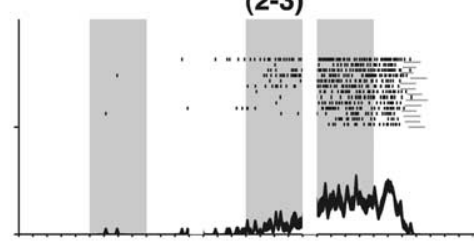

(3-4)

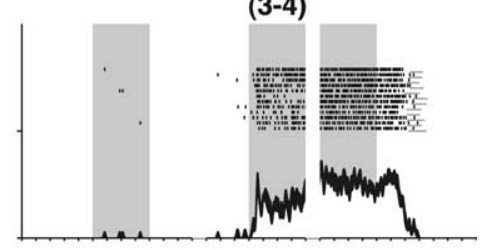

(4-5)

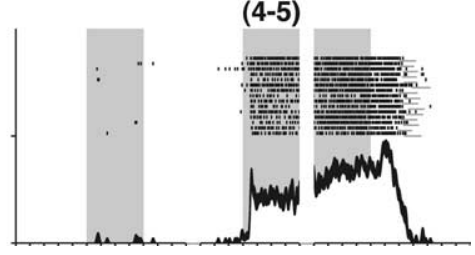

Figure 3. Neuronal activity reflecting a motor plan. In this display, neuronal activity is sorted according to the location of the target on the screen (Fig. 1C, T1 to T5), as well as to the instructions signaling a left or right future target. During the later part of the choice cue period, this neuron exhibited progressively greater activity as the target was placed in positions farther to the right. Nevertheless, the activity did not reflect what was specified by the instruction signals.

of individual neurons in $200 \mathrm{~ms}$ time bins during the task period preceding the onset of the GO signal. We classified each neuron into one of five categories by consecutively applying ANOVA to the data collected in the $200 \mathrm{~ms}$ bins: neurons selective only for the action plan, neurons selective for both the action plan and the choice cue location (mixed selectivity), neurons selective only for the choice cue location, neurons selective only for the motor plan, and neurons selective for none of these variables (see Materials and Methods). The results of this analysis are summarized as a time-dependent distribution in Figure 5 as a population and in Figure 6 for individual neurons. Before the appearance of the instruction cue, few neurons with selectivity for any of the variables were identified. After the presentation of the instruction cue, neuronal activity representing the action plan appeared quickly; in the second $200 \mathrm{~ms}$ bin after the onset of the instruction cue (101-300 ms after the instruction cue was presented), 219 of the 773 neurons (28\%) showed activity selective for the action plan. During the subsequent instructed delay period before the appearance of the choice cue, this type of selectivity was observed 
(Left)

T1

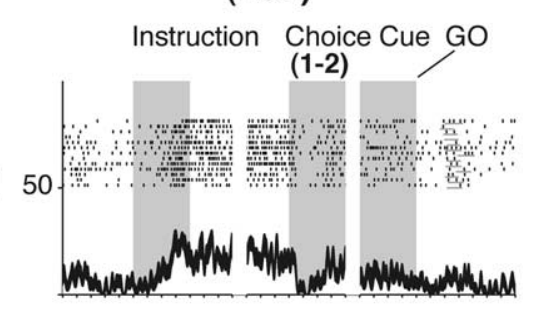

(2-3)

T2

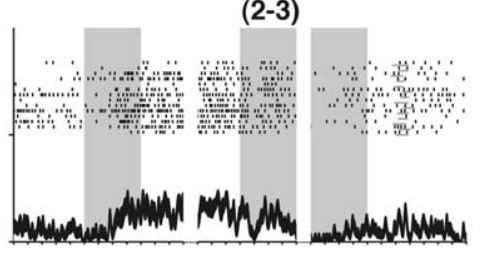

(3-4)

T3

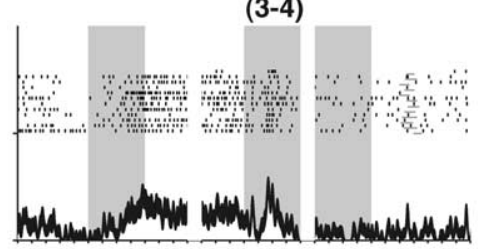

(4-5)

T4

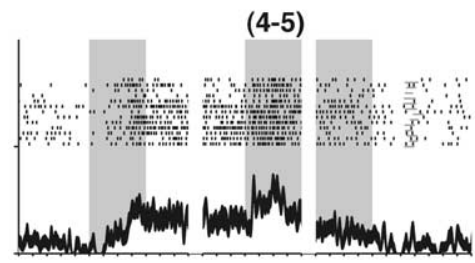

(5-6)

T5

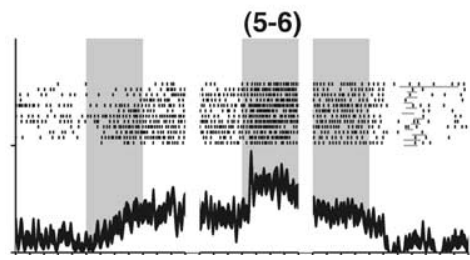

(Right)

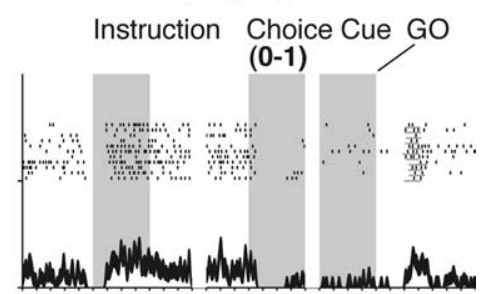

(1-2)

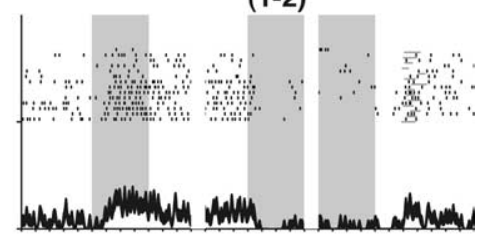

(2-3)

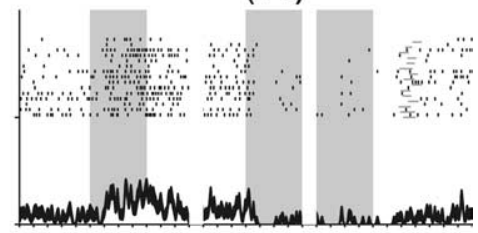

(3-4)

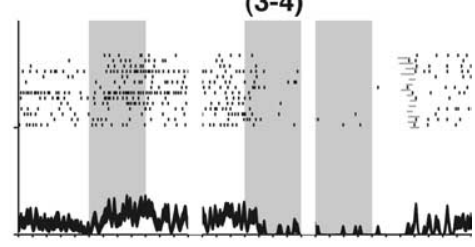

(4-5)

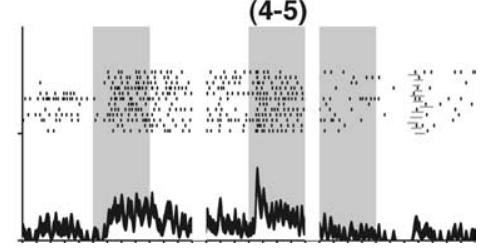

Figure 4. Neuronal activity reflecting two behavioral factors. This neuron was more active when the monkey was presented with instruction signals that indicated that the target was going to be on the left. Subsequently, when the choice cue appeared, the activity of this neuron changed as a function of two behavioral factors; the activity was greater if the target was located more to the right of the display or if the instruction indicated that the future target was going to be on the left. The display formats are the same as those used for Figure 3.

in $>24 \%$ of the task-related neurons. These results revealed that the activity of neurons in the PMd promptly reflected the action plan, and that the encoded information was maintained in these neurons until the choice cue was presented. However, the number of neurons in this category dropped quickly after the appearance of the choice cue. In the $200 \mathrm{~ms}$ bin (101-300 ms) just after the appearance of the choice cue, the number of neurons that only represented the action plan decreased to $80(10 \%$ of the neurons) from the 193 (25\%) that were identified in the $200 \mathrm{~ms}$ bin (301-100 ms) preceding the appearance of the choice cue.

However, the number of neurons that were selective for the location of the choice cue quickly increased; 90 neurons (12\%) were selective for both the choice cue location and the action plan. Another category of neurons that exclusively represented the choice cue location without representing the action plan was also identified $(n=34 ; 4 \%)$. In addition, 126 neurons $(16 \%)$ were only selective for the motor plan. During the subsequent delay period, which lasted between 1.5 and $2.5 \mathrm{~s}$, the activity selective for the motor plan was detected in $\sim 15 \%$ of the neu- rons, whereas the number of neurons reflecting both the action plan and the choice cue location decreased, dropping to $34(4 \%)$ at the end of the choice cue period. These results reflect the time course of the activity of PMd neurons representing the action plan, which were promptly replaced with the activity representing the motor plan.

Task phase-dependent changes in the selectivity of individual neurons for the action plan and motor plan

To investigate how the selectivity of individual neurons for the action plan and motor plan develop and decline during the behavioral task, we calculated a measure defined as $\mathrm{hp}^{2}$ to evaluate the strength of the effects of the independent variables on neuronal activity (see Materials and Methods). The $\mathrm{hp}^{2}$ was calculated for the data collected in four different $200 \mathrm{~ms}$ bins (corresponding to bins A-D in Fig. 5): 301-100 ms before the onset of the instruction cue (bin A), 301-100 ms before the onset of the choice cue (bin B), 101-300 ms after the onset of the choice cue (bin C), and at the end of the choice cue period (bin D;200 ms centered at the GO signal onset). This analysis was performed for most of the task-related neurons, with the exception of those classified as selective only for the choice cue location and those for which spikes were not detected during the analyzed period. At the end of the initial control period (Fig. $7 A$ ), the values of $h p^{2}$ remained small, indicating the absence of selective activity. At the end of the instructed delay period that followed the instruction cue (Fig. $7 B$ ), the data points were scattered along the $y$-axis, indicating that the individual neurons represented the action plan, but not the motor plan. Subsequently, during an early part of the choice cue period (Fig. $7 C$ ), the data points were spread along the $x$-axis, as well as the $y$-axis. Thus, during this period, both the action plan and the motor plan influenced the neuronal activity. Near the end of the choice cue period (Fig. 7D), the representation of the action plan further diminished, whereas the activity representing the motor plan was well maintained. This analysis indicated that the population of PMd neurons, as a whole, transformed the information about the action plan into a motor plan; this transition took place substantially during the early stages of the choice cue presentation.

Time course of development of the selectivity for each of the action plan, choice cue location, and motor plan: population analysis

We performed a detailed analysis of the temporal profile of selectivity for each of the action plan, choice cue location, and motor plan, by using the $200 \mathrm{~ms}$ bin data explained above. We first measured selectivity for the action plan for a population of neurons that were classified as action selective $(n=193)$. The data shown in Figure $8 \mathrm{~A}$ revealed that the action selectivity developed quickly after the appearance of the instruction cue, which was main- 
Table 1. Neurons classified based on the selectivity observed during the instructed-delay and choice cue periods

\begin{tabular}{|c|c|c|c|c|c|}
\hline \multirow[b]{2}{*}{ Selectivity during the instructed-delay period ${ }^{a}$} & \multicolumn{5}{|c|}{ Selectivity during the choice cue period } \\
\hline & Action plan & Action plan and choice cue location & Motor plan & Choice cue location & Nonselective \\
\hline Action plan $(n=193)$ & $35(18)$ & $61(19)$ & $29(41)$ & $14(2)$ & $54(113)$ \\
\hline Nonselective $(n=580)$ & $45(36)$ & $29(15)$ & $97(68)$ & $20(9)$ & $389(452)$ \\
\hline Total $(n=773)$ & $80(54)$ & $90(34)$ & $126(109)$ & $34(11)$ & $443(565)$ \\
\hline
\end{tabular}

Data refer to the number of neurons exhibiting selectivity to the four aspects of behavioral factors during the early phase of the choice cue period (101-300 ms after the cue onset). The data in parentheses indicate the data obtained during the late phase of the choice cue period ( 200 ms period centered at the GO signal onset).

${ }^{a}$ Selectivity during the $200 \mathrm{~ms}$ at the end of the instructed delay period (301-100 ms before the choice cue onset).

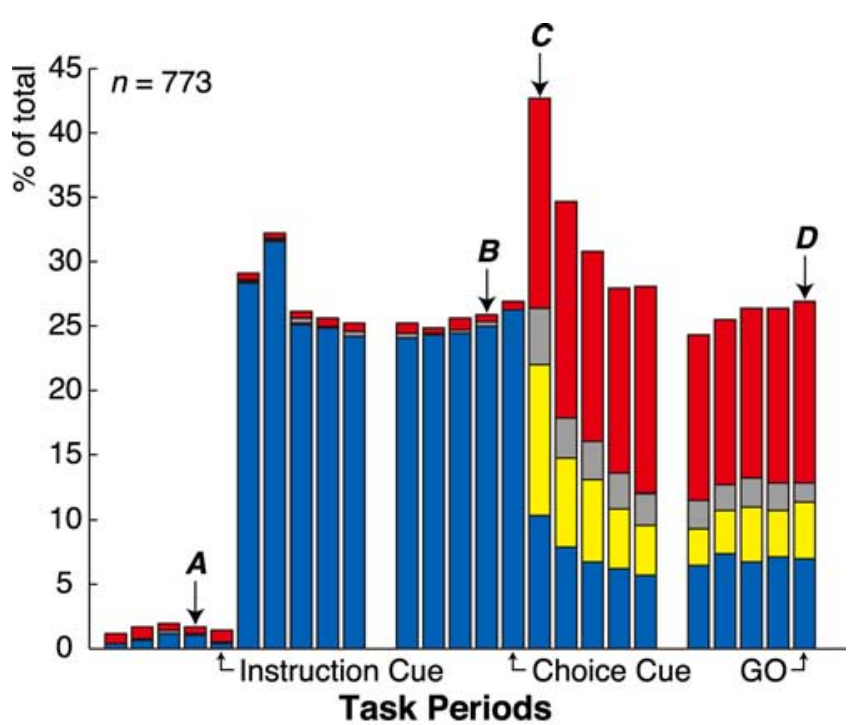

\begin{tabular}{|l|}
\hline Action Plan \\
Choice-Cue Location \\
Mixed Selectivity \\
(Action Plan \& Choice-Cue Location) \\
Motor Plan \\
\hline
\end{tabular}

Figure 5. Time-dependent distribution of neurons selective for each of the behavioral factors. During each $200 \mathrm{~ms}$ time bin in the task period, individual neurons were classified into one of five categories based on their specific selectivity for each of the behavioral factors. The results were then complied into a histogram. Different colors indicate the selectivity of the neurons for the action plan (blue), choice cue location (gray), action plan and choice cue location (yellow), and motor plan (red). One bin each is centered at the onsets of the instruction cue, choice cue, and $\mathrm{GO}$ signal. The arrows labeled with $\mathrm{A}, \mathrm{B}, \mathrm{C}$, and $\mathrm{D}$ indicate four bins for which values for a variable, $\mathrm{hp}^{2}$, were calculated for the data shown in Figure 7.

tained throughout the delay period but declined once the choice cue was presented. Figure $8 B$ show the selectivity for the choice cue location calculated for the population of neurons that were classified as selective only for the choice cue location $(n=34)$. The choice cue selectivity rapidly increased soon after its appearance and gradually declined during the choice cue period. Figure $8 D$ indicates that the selectivity for the motor plan appeared (among 109 neurons) soon after the onset of the choice cue $(<300 \mathrm{~ms})$, and was maintained throughout the choice cue period. In Figure $8 C$, we present the data for neurons classified as selective for both the action plan and the choice cue location (mixed selectivity, $n=90$ ). For this group of neurons, each of three categories of selectivity developed with variable time courses in the task periods. Soon after the appearance of the instruction cue, this group of neurons showed selectivity for the action plan. Once the choice cue was presented, selectivity for the choice cue and the motor plan developed promptly, whereas the action plan selectivity declined gradually.
Distribution of neurons with each class of selectivity during each task period

Examination of the task phase-dependent changes in the distribution of neurons exhibiting each class of selectivity (Table 1) revealed the following two findings. First, during the early choice cue period (Table 1, Fig. 6A), neurons with mixed selectivity (for both action plan and choice cue location) tended to be found more often among the group of neurons that had been selective for the action plan during the instructed delay period (61 vs 29; binomial test, $p=0.001$ ). This finding suggests that the integration of information for the action plan and the incoming choice cue information takes place mainly among neurons that exhibited action plan selectivity during the instructed delay period. In contrast, neurons selective only for the motor plan were found more often among neurons that had not been selective for the action plan ( 29 vs 97; binomial test, $p<0.0001$ ), suggesting that the motor plan was read out mainly by the group of neurons without the selectivity for the action plan during the delay period. Second, motor plan-selective neurons grew in number and were most prevalent $(n=109)$ during the late choice cue period (Table 1, Fig. $6 B$ ). These motor plan-selective neurons belonged to either action selective or nonselective neurons during the instructed delay period (indistinguishably distributed; 41 vs 68 ; binomial test, $p=0.0124$ ). Thus, at this behavioral stage of motor preparation immediately preceding the initiation of movements, motor plan selectivity developed among neurons regardless of whether each neuron participated in representing action plan or not during the delay period.

Relationship between the representations of the action plan and the motor plan in individual neurons: analysis for laterality As mentioned, a large number of the PMd neurons exhibited selectivity for both the action plan and the motor plan. Here, we examined relationship of the laterality of the former with the laterality of the latter. We analyzed neurons that were selective for the action plan, the action plan and the choice cue location, or the motor plan during the period 101-300 ms after the onset of the choice cue (Fig. 5) $(n=296)$. To assess the laterality of the neurons for the action plan, we calculated the difference in the firing rates that resulted from an instruction to reach for a future target on the right or the left. To measure the effect of the laterality of the motor plan, we calculated the difference between the activities measured when the monkey was planning to reach for a target on the left (T1 and T2) or the right (T4 and T5) of the screen. The results are shown in Figure 9 as a scatter plot. We did not observe a significant correlation between the laterality of the action plan selectivity and that of the motor plan selectivity $(r=0.0666 ; p=$ 0.2535 ), suggesting that these two values were independently encoded in individual neurons while the motor plan was developing. We reached the same conclusion by analyzing a subset of neurons whose activity was selective for both the action plan and the choice cue location (indicated with open circles in Fig. 9) $(n=$ 90; $r=0.1303 ; p=0.2210)$. 
A

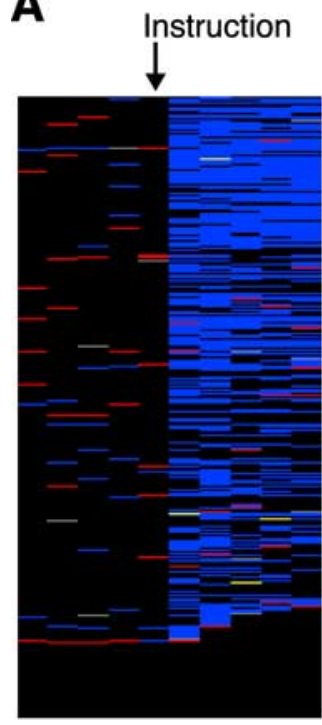

B

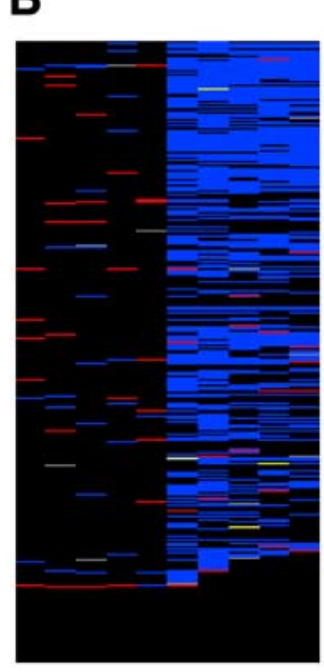

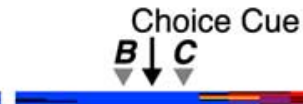
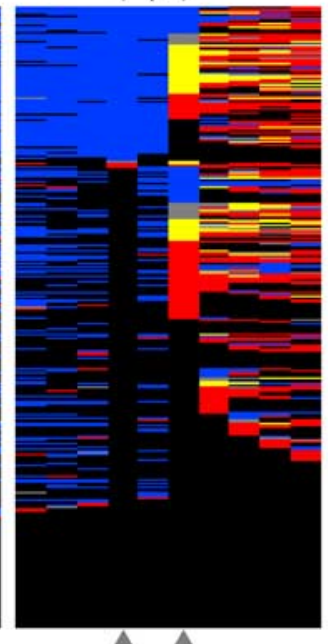

$B$

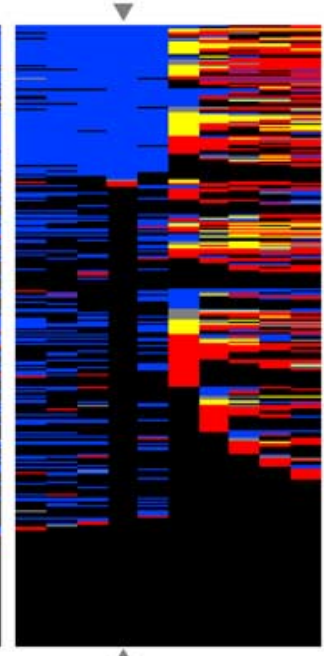

$\mathbf{A}$
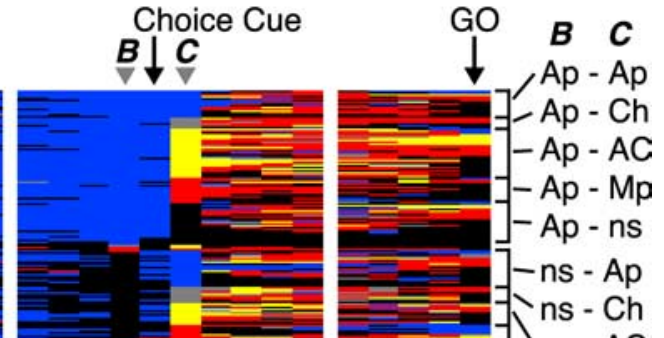

$=-\mathrm{Ap}-\mathrm{ns}$

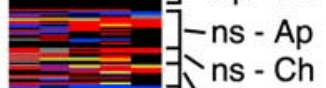

$=-1 \mathrm{~ns}-\mathrm{Ch}$

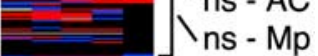

$=-\mathrm{ns}-\mathrm{ns}$

.

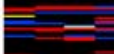

$=$

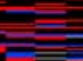

플
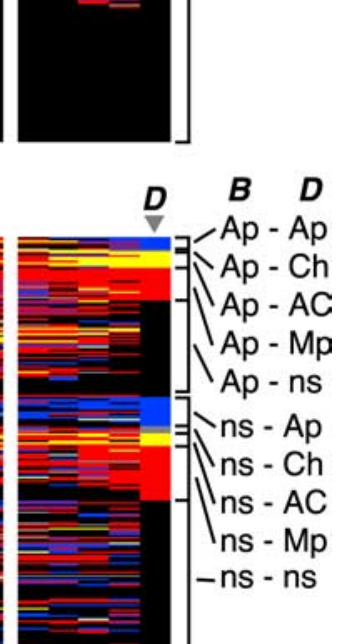

.
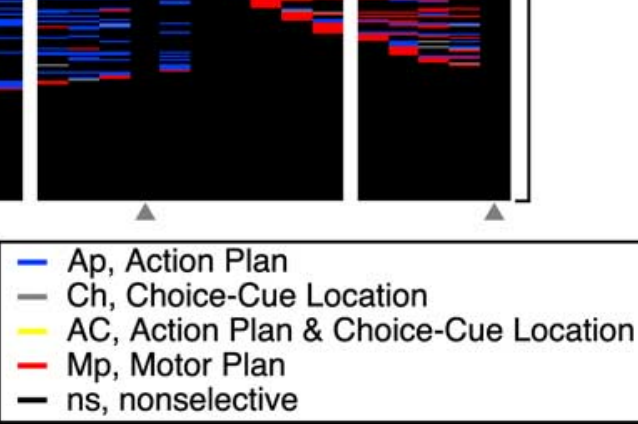

Figure 6. Time-dependent selectivity of each neuron for each of the behavioral factors. For each $200 \mathrm{~ms}$ time bin in the task period, properties of individual neurons were classified into one of five categories based on their specific selectivity for each of the behavioral factors. Activity profiles of individual neurons $(n=773)$ are then displayed consecutively; each row represents data for a single neuron. $\boldsymbol{A}$, Neurons were sorted according to the selectivity at the end of the instructed-delay period (marked with B) and just after the choice cue onset (marked with C). $\boldsymbol{B}$, Neurons were sorted according to the selectivity at the end of the instructeddelay period (marked with B) and at the $\mathrm{GO}$ signal onset (marked with D). Different colors indicate the selectivity of the neurons for the action plan (blue), choice cue location (gray), action plan and choice cue location (yellow), and motor plan (red). To the right of each panel, the selectivity of each neuron classified at the two 200 ms bins (labeled with B, C or B, D) is indicated. Ap, Activity selective for the action plan; $C h$, activity selective for the choice cue location; $A C$, activity selective for the action plan and choice cue location; Mp, activity selective for the motor plan; ns, nonselective activity.

\section{Localization of selective neurons}

Figure 10 shows the spatial distribution of neurons exhibiting four types of aforementioned selectivity in density maps drawn on the cortical surface. In each panel of this figure, we present the color-coded density of neurons exhibiting selectivity for the action plan (Fig. 10A), choice cue location (Fig. 10B), mixture of action plan and choice cue location (Fig. 10C), and motor plan (Fig. 10D) during three different task periods. At the end of the instructed delay period (left column), neurons selective for the instruction were found throughout the surveyed area of the caudal PMd but most densely close to the spur of the arcuate sulcus. During the early (middle column) and late (right column) choice cue periods, the four classes of selectivity were distributed throughout the caudal PMd. The density of neurons exhibiting each class of selectivity localized in the rostral and caudal one-half of the sampled area did not differ significantly ( $p>$ $0.05, \chi^{2}$ test).

\section{Possible influence of eye positions on} neuronal activity

The monkeys were required to gaze at a fixation point for $1.2 \mathrm{~s}$ before the appearance of the instruction cue and the choice cue. During these periods, the gaze was maintained within $1.0^{\circ}$ from the fixation point. Thus, it was unlikely that neuronal selectivity for the action plan, observed during the instructed delay period, was attributable to differences in the gaze direction. Subsequently, while the choice cue was on the screen for the period of 1.5-2.5 $\mathrm{s}$, the saccadic eye movements were made $6.1 \pm 2.3($ mean $\pm \mathrm{SD})$ times for monkey 1 and $5.8 \pm 1.8$ times for monkey 2 . To examine the influence of eye positions on neuronal activity, we first calculated averaged values of eye positions and spike rates during each $200 \mathrm{~ms}$ interval in the entire task period. The values of eye positions were then allocated to five compartments $\left(11 \times 11^{\circ}\right.$; centered at each of the five targets on the screen) and one additional compartment that covered the entire space outside of the five compartments. We, then, calculated a variable $\mathrm{hp}^{2}$ (see Materials and Methods) using a subset of the data set when the averaged eye position was in one of the five compartments covering the targets. In $99 \%$ of the cases, the $\mathrm{hp}^{2}$ values were $<0.2000(0.0439 \pm$ 0.0420 , mean \pm SD). Together with a fact that we sampled neuronal activity from the caudal part of the PMd where ICMS did not evoke saccadic eye movements, we interpreted that the effect of eye positions on neuronal activity was insignificant.

\section{Discussion}

We have shown that the activity of neurons in the PMd initially reflected a virtual action plan associated with a visual cue that represented a nonvisible potential target. Subsequently, when a pair of choice cues was provided, the information about the spatial position of the choice cue was rapidly combined with the information instructing the selection of the target, resulting in the development of a representation of a motor plan in PMd neurons, which eventually replaced the representation of the virtual action plan. The activity 


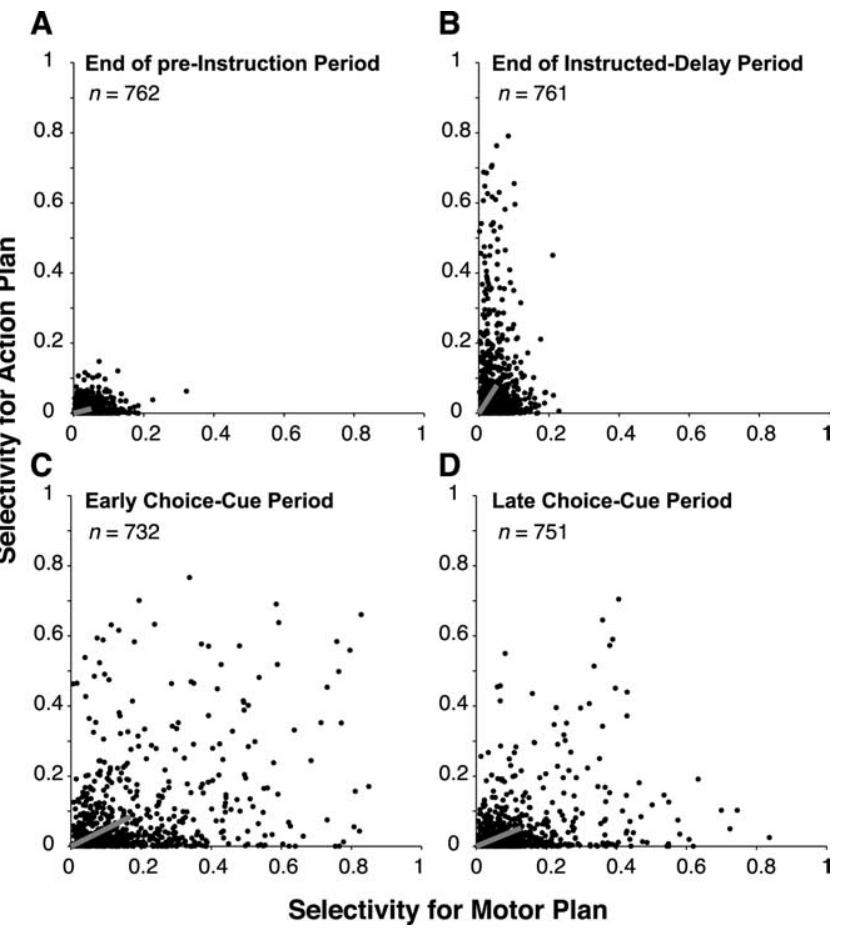

Figure 7. Comparing the selectivity of individual neurons using four behavioral epochs. To examine the behavioral epoch-dependent changes in the behavioral selectivity of individual neurons, the selectivity for the action plan was plotted against the selectivity for the motor plan during the following four epochs in the behavioral task: at the end of the preinstruction period immediately before the onset of the instruction cue (301-100 ms before its appearance; $n=$ 762) $(A)$; during a late part of the instructed delay period (301-100 ms before the appearance of the choice cue; $n=761$ ) (B); during an early part of the choice cue period (101-300 ms after the appearance of choice cue; $n=732$ ) (C); and during a late part of the choice cue period ( $200 \mathrm{~ms}$ centered at the $G 0$ signal onset; $n=751)$ (D). Behavioral selectivity refers to a statistical measure $\left(\mathrm{hp}^{2}\right)$ calculated using the data obtained during $200 \mathrm{~ms}$ time bins centered at four different behavioral epochs. The time bins used to present data in $\boldsymbol{A}-\boldsymbol{D}$ correspond to the time bins indicated with labels $A-D$ in Figure 5 . The gray-colored line in each panel points to the mean values of each variable of the analyzed neurons.

representing both the action plan and the motor plan appeared with a fairly short latency $(<300 \mathrm{~ms})$ after the instruction or choice cues. We also found that some PMd neurons first exhibited activity representing the virtual action plan, which changed into activity representing both the action plan and the choice cue location after the appearance of the choice cue, suggesting that these neurons contribute to the transformation of information during the behavioral task. Moreover, while the motor plan was developing, the laterality of the selectivity for the virtual action plan represented in each PMd neuron did not correlate with the laterality of the selectivity for the direction that the monkeys reached for the target. This observation makes it unlikely that the neuronal selectivity for the virtual action plan was markedly influenced by a bias for spatial attention, covert motor preparation, or motor preparation in object-centered frame of reference (see also supplemental Discussion 1 and 2, available at www.jneurosci.org as supplemental material). We concluded that the PMd contributes to a neural network that underlies the implementation of a motor plan after integrating two sets of inputs that represent a virtual action plan and information provided by a choice cue. A conceptual framework of this view can be visualized as in Figure 11, where the workings of the hypothetical network are proposed (for possible operation of the network, see supplemental Discussion 3, available at www.jneurosci.org as supplemental material).

\section{A Neurons selective for Action Plan}

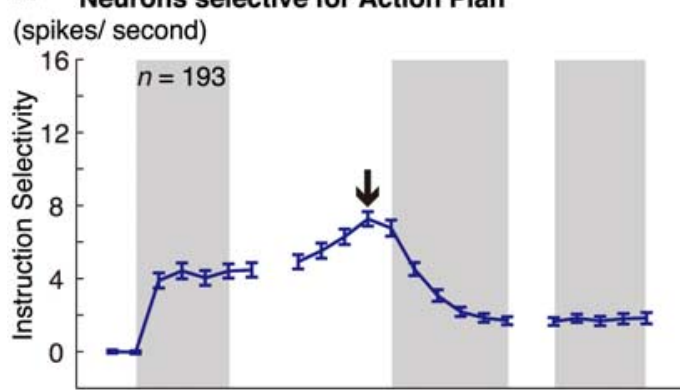

\section{B Neurons selective for Choice-Cue Location}

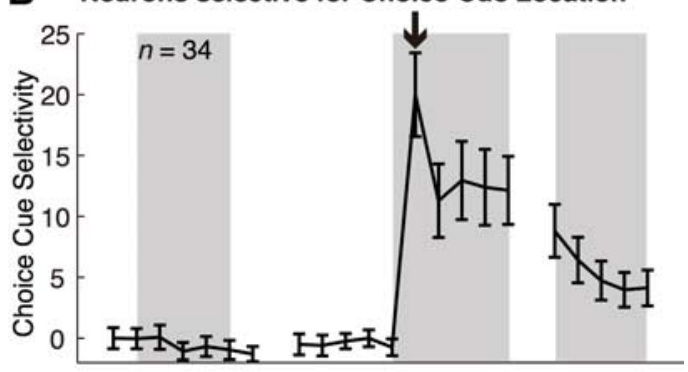

C Neurons with Mixed Selectivity
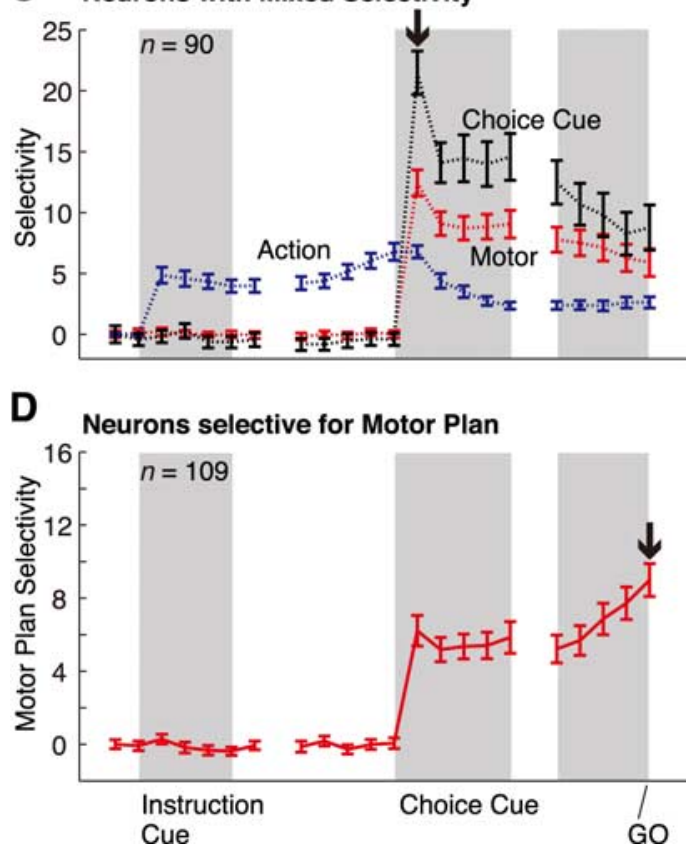

Figure 8. Temporal profiles of the magnitude of firing selectivity calculated for the population of neurons classified into four categories. $\boldsymbol{A}$, Selectivity for the action plan, calculated as the absolute difference in firing rates responding to the left and right instruction. Data were analyzed for neurons that were classified as action selective during the late part of the instructeddelay period (301-100 ms before the choice cue onset, indicated by the arrow; $n=193$ ). The selectivity in $A-D$ is expressed as a relative increase compared with the control period (301-100 ms before the instruction cue). In $\boldsymbol{A}-\boldsymbol{D}$, the ordinate represents the selectivity in the firing rate (in spikes/second). $\boldsymbol{B}$, Selectivity for the choice cue location, calculated as the differences in activity when the choice cue appeared at the two locations leading to the highest and lowest activity. This analysis was applied to neurons that were selective only for the choice cue location during the early part of the choice cue period (101-300 ms after the choice cue onset, indicated by the arrow; $n=34$ ). C, Selectivity for the action plan (blue), choice cue location (black), and motor plan (red) among neurons that exhibited the mixed type of selectivity during the early part of the choice cue period (indicated by the arrow; $n=90$ ). D, Selectivity for the motor plan, calculated as the differences in activity when the reach target was in the two positions leading to the highest and lowest activity. This analysis was applied for the neurons that showed selectivity only for the motor plan during the late part of the choice cue period (200 ms period centered at the $\mathrm{G} 0$ signal onset, indicated by the arrow; $n=109$ ). 


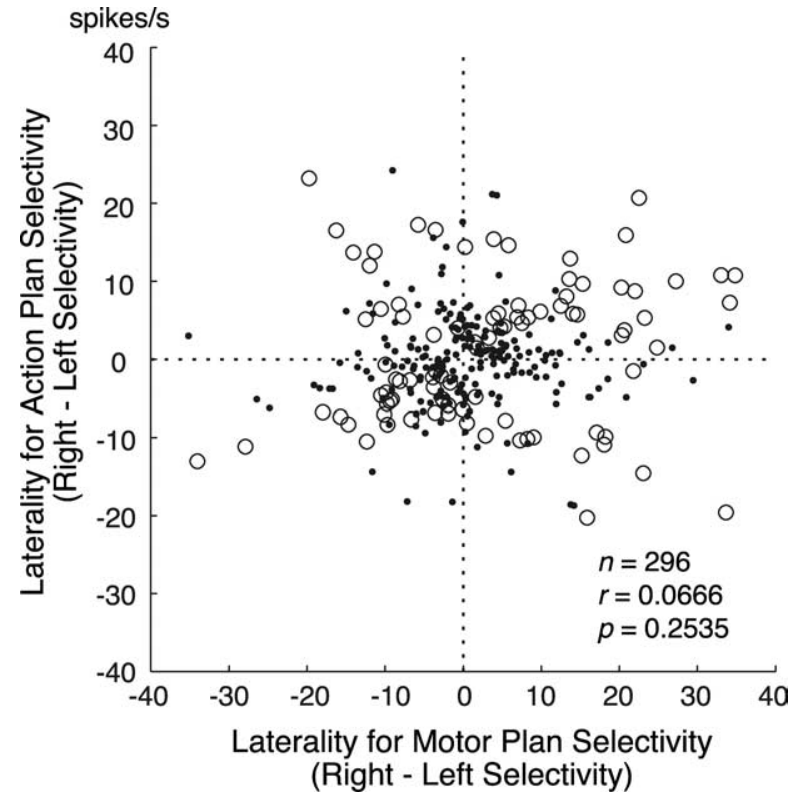

Figure 9. Relationship of the laterality between the action plan-selective activity and the motor plan-selective activity of individual neurons. The laterality of the neuronal activity selective for the action plan is plotted along the $y$-axis, whereas the laterality of the motor plan is plotted along the $x$-axis (positive and negative values denote rightward and leftward selectivity, respectively). Each dot represents data for an individual neuron. For this analysis, we included neurons that exhibited selectivity for the action plan, the action plan and the choice cue location, or the motor plan during the 101-300 ms period after the choice cue onset ( $n=296$ ). The correlation coefficient was $0.0666(p=0.2535)$. Application of the same analysis to a subset of neurons whose activity was selective for the action plan and the choice cue location (indicated with open circles; $n=90)$ again did not reveal significant correlation $(r=0.1303$; $p=0.2210)$.

Previous reports have established that the PM plays a role in preparing forthcoming movements (Wise, 1985). The activities of PM neurons are also known to reflect parameters of intended movements, such as direction and amplitude (Fu et al., 1993; Kurata, 1993). The directional selectivity of the neuronal activity depended on the workspace during reaching movements (Caminiti et al., 1991) or on the posture of the reaching arm (Scott et al., 1997). In the present study, $\sim 15 \%$ of the task-related neurons exhibited activity selective for the target that the monkey planned to select (Fig. 3). This finding indicates that, as far as motor preparation or planning is concerned, our results are in general agreement with previous reports.

However, previous reports suggest that the PM is involved in regulating motor behaviors at stages before the parameters of the planned movements are determined. Neurons in the ventral aspect of the PM (PMv) were found to encode the direction of movement in space independent of the forearm posture (Kakei et al., 2001). Schwartz et al. (2004) showed that some PMv neurons encoded a movement trajectory in a perceived visual space rather than representing the actual arm movements. Ohbayashi et al. (2003) showed that the PMd neurons maintained information about spatial cues before it was converted into a sequential motor behavior. PMd neurons were also found to represent the potential direction of reach targets before a reach target was specified (Bastian et al., 2003; Cisek and Kalaska, 2005). In separate line of studies, PMd neurons were shown to retrieve and integrate two sets of information about arm use or target location for planning reaching movements (Hoshi and Tanji, 2000, 2006; Beurze et al., 2007).

Another set of experiments revealed the role of the PM in
A Action Plan Selectivity

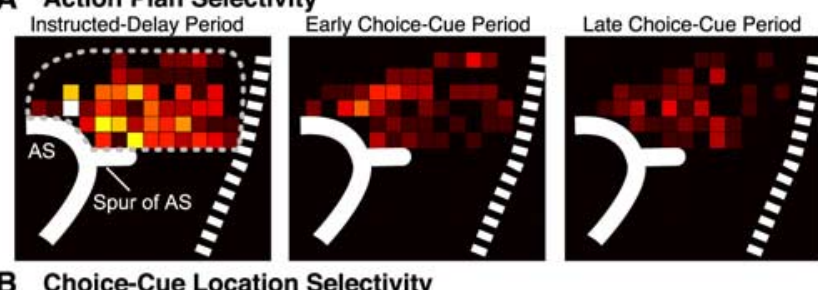

B Choice-Cue Location Selectivity
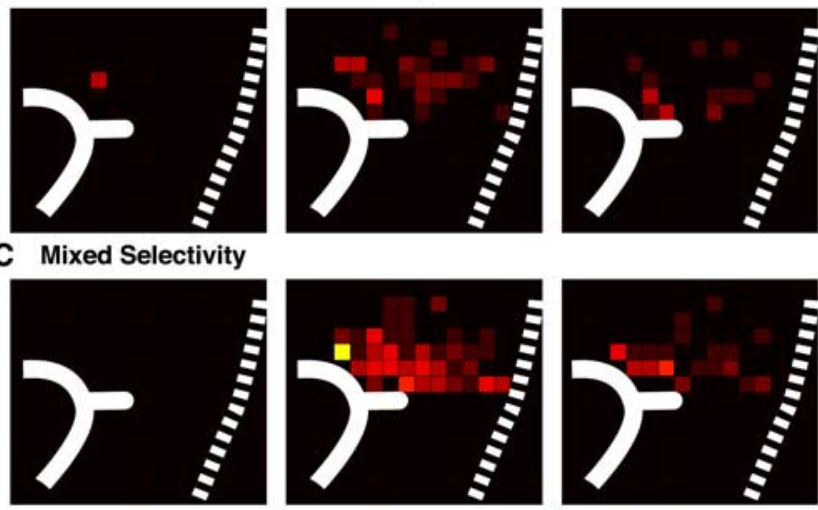

D Motor Plan Selectivity

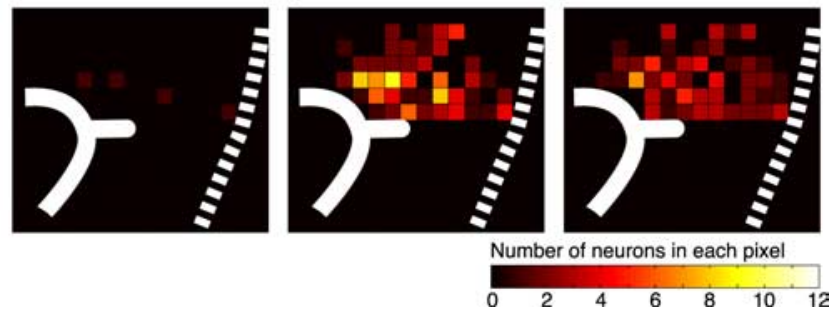

Figure 10. Density map of PM neurons with task selectivity drawn on cortical surface. The number of neurons exhibiting selectivity for the action plan $(\boldsymbol{A})$, choice cue location $(\boldsymbol{B})$, both action plan and choice cue location (mixed selectivity) $(\boldsymbol{C})$, and motor plan $(\boldsymbol{D})$ is summed in each pixel $(1 \times 1 \mathrm{~mm})$ subdividing the recorded area, and is color-coded to construct a density map. Data from the two animals were overlaid with reference to the genu of the arcuate sulcus. Three data sets obtained during the end of the instructed-delay period (left column), during the early (middle column) and late (right column) choice cue period, are illustrated separately. The three time periods correspond to the $200 \mathrm{~ms}$ bins labeled B, C, and D in Figure 5. The color codes are indicated with a scale at the bottom right. The solid line depicts the location of the arcuate sulcus (AS) with its spur. The broken line indicates the border between the premotor cortex and the primary motor cortex. The dotted line in the top left panel encloses the recorded area.

associating visual signals with appropriate movements (Halsband and Passingham, 1982; Petrides, 1982; Halsband and Freund, 1990; Passingham, 1993; Toni et al., 1999). Petrides (1986) revealed that PMd lesions affected the selection of symmetrically reinforced go and no-go decisions, suggesting that the lesion impaired a process associating visual signals with one of multiple representations of goal-directed actions. This aspect of the involvement of the PM in sensory-motor mapping was substantiated by studies examining single-cell activity during tasks that required the association of visual signals with motor behaviors (Mitz et al., 1991; Boussaoud and Wise, 1993; Kurata and Hoffman, 1994; Chen and Wise, 1995; Wise and Murray, 2000). In these studies, however, visual signals were directly associated with motor targets or the parameters of intended movements.

PMd neurons have also been shown to reflect a behavioral rule for the initiation of a movement (White and Wise, 1999; Wallis and Miller, 2003). Wallis and Miller (2003) trained monkeys in a task to perform a delayed match-to-sample task, and the monkeys had to make either go or no-go responses according to either a same or different rule. They found that the decision rule was 


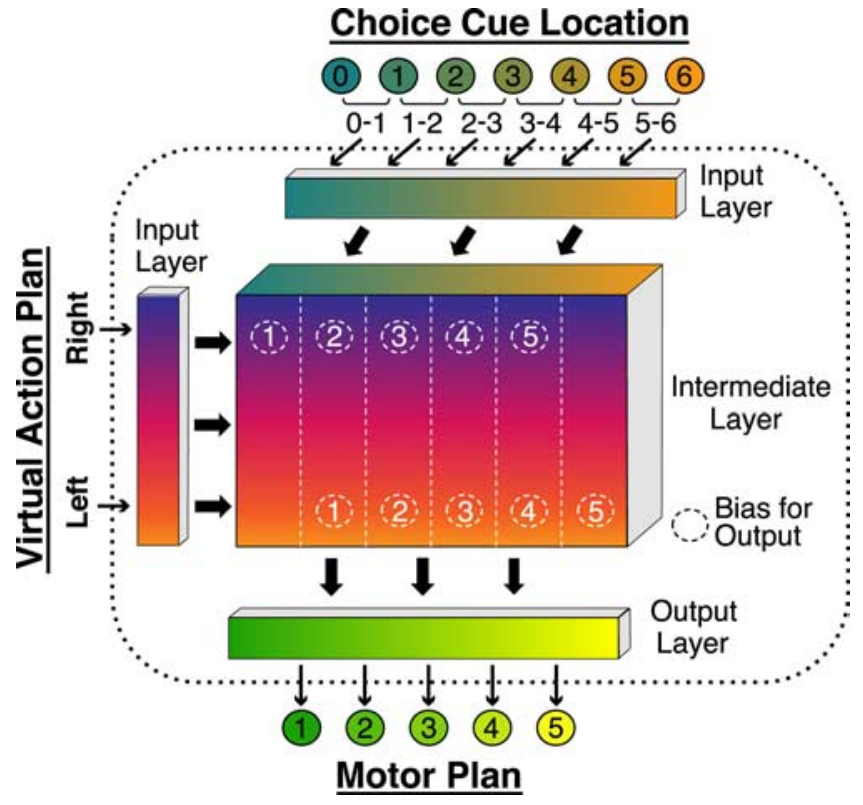

Figure 11. A schematic drawing of the main findings in this study. In this diagram, the premotor cortex is conceptualized as a network that includes two input layers, an intermediate layer, and an output layer. The two input layers retrieve information about the virtual action plan and the choice cue location, whereas the output layer transmits signals about the motor plan. The intermediate layer integrates the two sets of information provided by the two input layers and transforms them into information that can be used by the output layer.

represented more strongly in the PMd than in the prefrontal cortex. Because the response was predetermined and invariant (either release or not to release), it was not necessary to generate information about an action to be planned at an abstract level before that information was converted to actual motor plan. In the present study, we explored the role of the PM in a different aspect of the cognitive control of behavior that links the acquisition of an action plan with the formation of motor plan. A critical factor here is the representation of a virtual action plan in the absence of motor targets. What we intend to present in this study is the representation of right/left at an abstract level in the PM that, at a later behavioral stage, is transformed into information specifying the direction of motor output along the right-left axis.

Rizzolatti et al. (1987) reported that PMv neurons represented goal-directed actions; neuronal activity was similar regardless of the motor effectors (i.e., the body part used), such that it reflected the aim rather than the specific details of the movements. Neurons in the same area were found to discharge when an animal was observing a motor act done by others, as well as when the same motor act was executed by the animal itself (Gallese et al., 1996; Rizzolatti and Craighero, 2004). Cisek and Kalaska (2004) reported that neurons in the PMd exhibited similar activities regardless of whether a rule-based motor act was executed by the animal itself or by others. In these studies, neuronal discharges were triggered by directly observing the target of intended movements or by observing the motor action being performed toward a motor target. In contrast, the virtual-action-selective activity observed in the present study was initiated when an instruction specified a motor act, whereas no information was available to specify the motor target.

For the present behavioral task, the information specifying the virtual-action plan was constructed out of instructions containing visual objects with specific colors and shapes. It should be noted, however, that we did not find evidence that the PMd represented the identities of the visual stimuli. Several lines of evidence suggest that the prefrontal cortex is involved in this process (for review, see Miller, 2000; Murray et al., 2000; Bunge et al., 2005; Tanji and Hoshi, 2008). Lesions of the prefrontal cortex caused deficits in determining relationships between a visual object and a motor response (Wang et al., 2000; Bussey et al., 2001). At the single-cell level, lateral prefrontal neurons were found to contribute to the association between a visual object and a motor response (Asaad et al., 1998). Genovesio et al. (2005) revealed that the process of visuomotor association is strongly influenced by the behavioral strategy (i.e., repeat stay and change shift). Niki (1974) examined the activity of prefrontal neurons while monkeys were instructed to select the right or left cued target, and found that $18 \%$ of them responded to the relative position of the targets. These types of activity in the prefrontal cortex may contribute to the PM activity observed during the choice cue period in this study, which exhibited selectivity for the right or left component of the choice cue. Olson and coworkers (Olson and Gettner, 1995; Tremblay et al., 2002; Moorman and Olson, 2007) reported that neurons in the supplementary eye field (SEF) were selective for the planning of eye movements to either end of the reference objects. Furthermore, the activities of SEF neurons were found to reflect saccade directions during a delay period, even if the direction was specified by chromatic cues (Olson and Gettner, 1999). Chen and Wise (1996) revealed the involvement of the SEF in determining the direction of saccadic eye movements based on pictorial cues. Together, these previous reports point to the participation of the rostral frontal cortex in retrieving visual-object information and in transforming it into information representing action that is not directly bound to physical movement parameters. The information could then be transferred via corticocortical connections to the PM ( $\mathrm{Lu}$ et al., 1994; Luppino et al., 2003; Takada et al., 2004), where it is further transformed into information representing a motor plan.

In summary, our current results point to an aspect of the functional role played by PMd neurons in representing an abstract description of an action and in transforming it into information required to generate a motor plan.

\section{References}

Asaad WF, Rainer G, Miller EK (1998) Neural activity in the primate prefrontal cortex during associative learning. Neuron 21:1399-1407.

Bastian A, Schöner G, Riehle A (2003) Preshaping and continuous evolu-

tion of motor cortical representations during movement preparation. Eur J Neurosci 18:2047-2058.

Beurze SM, de Lange FP, Toni I, Medendorp WP (2007) Integration of target and effector information in the human brain during reach planning. J Neurophysiol 97:188-199.

Boettiger CA, D’Esposito M (2005) Frontal networks for learning and executing arbitrary stimulus-response associations. J Neurosci 25:2723-2732.

Boussaoud D, Wise SP (1993) Primate frontal cortex: effects of stimulus and movement. Exp Brain Res 95:28-40.

Bruce CJ, Goldberg ME, Bushnell MC, Stanton GB (1985) Primate frontal eye fields. II. Physiological and anatomical correlates of electrically evoked eye movements. J Neurophysiol 54:714-734.

Bunge SA, Wallis JD, Parker A, Brass M, Crone EA, Hoshi E, Sakai K (2005) Neural circuitry underlying rule use in humans and nonhuman primates. J Neurosci 25:10347-10350.

Bussey TJ, Wise SP, Murray EA (2001) The role of ventral and orbital prefrontal cortex in conditional visuomotor learning and strategy use in rhesus monkeys (Macaca mulatta). Behav Neurosci 115:971-982.

Caminiti R, Johnson PB, Galli C, Ferraina S, Burnod Y (1991) Making arm movements within different parts of space: the premotor and motor cortical representation of a coordinate system for reaching to visual targets. J Neurosci 11:1182-1197.

Caminiti R, Ferraina S, Mayer AB (1998) Visuomotor transformations: early cortical mechanisms of reaching. Curr Opin Neurobiol 8:753-761. 
Canavan AG, Nixon PD, Passingham RE (1989) Motor learning in monkeys (Macaca fascicularis) with lesions in motor thalamus. Exp Brain Res 77:113-126.

Chen LL, Wise SP (1995) Neuronal activity in the supplementary eye field during acquisition of conditional oculomotor associations. J Neurophysiol 73:1101-1121.

Chen LL, Wise SP (1996) Evolution of directional preferences in the supplementary eye field during acquisition of conditional oculomotor associations. J Neurosci 16:3067-3081.

Cisek P, Kalaska JF (2004) Neural correlates of mental rehearsal in dorsal premotor cortex. Nature 431:993-996.

Cisek P, Kalaska JF (2005) Neural correlates of reaching decisions in dorsal premotor cortex: specification of multiple direction choices and final selection of action. Neuron 45:801-814.

Crammond DJ, Kalaska JF (1994) Modulation of preparatory neuronal activity in dorsal premotor cortex due to stimulus-response compatibility. J Neurophysiol 71:1281-1284.

Deiber MP, Wise SP, Honda M, Catalan MJ, Grafman J, Hallett M (1997) Frontal and parietal networks for conditional motor learning: a positron emission tomography study. J Neurophysiol 78:977-991.

di Pellegrino G, Wise SP (1993) Visuospatial versus visuomotor activity in the premotor and prefrontal cortex of a primate. J Neurosci 13:1227-1243.

Eliassen JC, Souza T, Sanes JN (2003) Experience-dependent activation patterns in human brain during visual-motor associative learning. J Neurosci 23:10540-10547.

Fu QG, Suarez JI, Ebner TJ (1993) Neuronal specification of direction and distance during reaching movements in the superior precentral premotor area and primary motor cortex of monkeys. J Neurophysiol 70:2097-2116.

Fujii N, Mushiake H, Tanji J (2000) Rostrocaudal distinction of the dorsal premotor area based on oculomotor involvement. J Neurophysiol 83:1764-1769.

Gallese V, Fadiga L, Fogassi L, Rizzolatti G (1996) Action recognition in the premotor cortex. Brain 119:593-609.

Genovesio A, Brasted PJ, Mitz AR, Wise SP (2005) Prefrontal cortex activity related to abstract response strategies. Neuron 47:307-320.

Grafton ST, Fagg AH, Arbib MA (1998) Dorsal premotor cortex and conditional movement selection: a PET functional mapping study. J Neurophysiol 79:1092-1097.

Halsband U, Freund HJ (1990) Premotor cortex and conditional motor learning in man. Brain 113:207-222.

Halsband U, Passingham RE (1982) The role of premotor and parietal cortex in the direction of action. Brain Res 240:368-372.

Halsband U, Passingham RE (1985) Premotor cortex and the conditions for movement in monkeys (Macaca fascicularis). Behav Brain Res 18:269-277.

Hoshi E, Tanji J (2000) Integration of target and body-part information in the premotor cortex when planning action. Nature 408:466-470.

Hoshi E, Tanji J (2006) Differential involvement of neurons in the dorsal and ventral premotor cortex during processing of visual signals for action planning. J Neurophysiol 95:3596-3616.

Hoshi E, Tanji J (2007) Distinctions between dorsal and ventral premotor areas: anatomical connectivity and functional properties. Curr Opin Neurobiol 17:234-242.

Kakei S, Hoffman DS, Strick PL (2001) Direction of action is represented in the ventral premotor cortex. Nat Neurosci 4:1020-1025.

Kalaska JF, Crammond DJ (1992) Cerebral cortical mechanisms of reaching movements. Science 255:1517-1523.

Kurata K (1993) Premotor cortex of monkeys: set- and movement-related activity reflecting amplitude and direction of wrist movements. J Neurophysiol 69:187-200.

Kurata K, Hoffman DS (1994) Differential effects of muscimol microinjection into dorsal and ventral aspects of the premotor cortex of monkeys. J Neurophysiol 71:1151-1164.

Lu MT, Preston JB, Strick PL (1994) Interconnections between the prefrontal cortex and the premotor areas in the frontal lobe. J Comp Neurol 341:375-392.

Luppino G, Rozzi S, Calzavara R, Matelli M (2003) Prefrontal and agranular cingulate projections to the dorsal premotor areas F2 and F7 in the macaque monkey. Eur J Neurosci 17:559-578.

Matelli M, Luppino G, Rizzolatti G (1985) Patterns of cytochrome oxidase activity in the frontal agranular cortex of the macaque monkey. Behav Brain Res 18:125-136.

Miller EK (2000) The prefrontal cortex and cognitive control. Nat Rev Neurosci 1:59-65.

Mitz AR, Godschalk M, Wise SP (1991) Learning-dependent neuronal activity in the premotor cortex: activity during the acquisition of conditional motor associations. J Neurosci 11:1855-1872.

Moorman DE, Olson CR (2007) Combination of neuronal signals representing object-centered location and saccade direction in macaque supplementary eye field. J Neurophysiol 97:3554-3566.

Murray EA, Bussey TJ, Wise SP (2000) Role of prefrontal cortex in a network for arbitrary visuomotor mapping. Exp Brain Res 133:114-129.

Mushiake H, Inase M, Tanji J (1991) Neuronal activity in the primate premotor, supplementary, and precentral motor cortex during visually guided and internally determined sequential movements. J Neurophysiol 66:705-718.

Niki H (1974) Prefrontal unit activity during delayed alternation in the monkey. II. Relation to absolute versus relative direction of response. Brain Res 68:197-204.

Ohbayashi M, Ohki K, Miyashita Y (2003) Conversion of working memory to motor sequence in the monkey premotor cortex. Science 301:233-236.

Olson CR, Gettner SN (1995) Object-centered direction selectivity in the macaque supplementary eye field. Science 269:985-988.

Olson CR, Gettner SN (1999) Macaque SEF neurons encode objectcentered directions of eye movements regardless of the visual attributes of instructional cues. J Neurophysiol 81:2340-2346.

Passingham RE (1993) The frontal lobes and voluntary action. Oxford: Oxford UP.

Petrides M (1982) Motor conditional associative-learning after selective prefrontal lesions in the monkey. Behav Brain Res 5:407-413.

Petrides M (1986) The effect of periarcuate lesions in the monkey on the performance of symmetrically and asymmetrically reinforced visual and auditory go, no-go tasks. J Neurosci 6:2054-2063.

Picard N, Strick PL (2001) Imaging the premotor areas. Curr Opin Neurobiol 11:663-672.

Rizzolatti G, Craighero L (2004) The mirror-neuron system. Annu Rev Neurosci 27:169-192.

Rizzolatti G, Gentilucci M, Fogassi L, Luppino G, Matelli M, Ponzoni-Maggi $S$ (1987) Neurons related to goal-directed motor acts in inferior area 6 of the macaque monkey. Exp Brain Res 67:220-224.

Schwartz AB, Moran DW, Reina GA (2004) Differential representation of perception and action in the frontal cortex. Science 303:380-383.

Scott SH, Sergio LE, Kalaska JF (1997) Reaching movements with similar hand paths but different arm orientations. II. Activity of individual cells in dorsal premotor cortex and parietal area 5. J Neurophysiol 78:2413-2426.

Takada M, Nambu A, Hatanaka N, Tachibana Y, Miyachi S, Taira M, Inase M (2004) Organization of prefrontal outflow toward frontal motor-related areas in macaque monkeys. Eur J Neurosci 19:3328-3342.

Tanji J, Hoshi E (2008) Role of the lateral prefrontal cortex in executive behavioral control. Physiol Rev 88:37-57.

Toni I, Schluter ND, Josephs O, Friston K, Passingham RE (1999) Signal-, set- and movement-related activity in the human brain: an event-related fMRI study. Cereb Cortex [Erratum (1999) 9:196] 9:35-49.

Toni I, Rushworth MF, Passingham RE (2001) Neural correlates of visuomotor associations. Spatial rules compared with arbitrary rules. Exp Brain Res 141:359-369.

Tremblay L, Gettner SN, Olson CR (2002) Neurons with object-centered spatial selectivity in macaque SEF: do they represent locations or rules? J Neurophysiol 87:333-350.

Wallis JD, Miller EK (2003) From rule to response: neuronal processes in the premotor and prefrontal cortex. J Neurophysiol 90:1790-1806.

Wang M, Zhang H, Li BM (2000) Deficit in conditional visuomotor learning by local infusion of bicuculline into the ventral prefrontal cortex in monkeys. Eur J Neurosci 12:3787-3796.

White IM, Wise SP (1999) Rule-dependent neuronal activity in the prefrontal cortex. Exp Brain Res 126:315-335.

Wise SP (1985) The primate premotor cortex: past, present, and preparatory. Annu Rev Neurosci 8:1-19.

Wise SP, Murray EA (2000) Arbitrary associations between antecedents and actions. Trends Neurosci 23:271-276. 